\title{
ROTATIONAL ENERGY TRANSFER IN MOLECULAR COLLISIONS AND PARAMETERS OF POWER-GAP "LAW"
}

\author{
P.M. Agrawal and S. Tilwankar* \\ School of Studies in Physics, Vikram University \\ Ujjain MP 456010, India
}

(Received June 5, 1997)

\begin{abstract}
The quantum mechanical integral inelastic cross-sections for the rotation transitions in a diatomic molecule with an atom were computed and analysed by using the empirical power-gap "law" over a wide range of collision energy. A well-known parameter, $|\Delta E|^{*}$, of the power-gap "law" was estimated by observing a rapid fall of cross-sections for the rotational energy transfer, $|\Delta E| \geq|\Delta E|^{*}$. It was found that $|\Delta E|^{*}$ corresponds to classical limit of maximum amount of rotational energy transfer permissible by the angular momentum conservation constraints and the hard ellipsoid potential model provided the hard ellipsoid potential surface is represented by the classical turning point surface of the real potential employed in the computation of cross-sections. Such an agreement is shown to be useful in the determination of the difference of major and minor axes of the intermolecular-potential ellipsoid from the knowledge of the cross-sections and the power-gap "law".

PACS numbers: $34.50 . \mathrm{Ez}, 34.50 .-\mathrm{s}$
\end{abstract}

\section{Introduction}

One of the important outcomes of the recent experimental and computational efforts in studying rotational energy transfer (RET) in molecular collisions is the evolution of empirical scaling and fitting laws which attempt to fit the entire matrix of integral inelastic cross-sections (IICS), $\sigma\left(j_{\mathrm{i}} \rightarrow j_{\mathrm{f}}\right.$ ), and rate constants in terms of a few parameters. Considerable attention has been focussed on the power-gap (PG) law [1-5] for fitting of RET data. Such a fitting of IICS data is expected to lead to a better understanding of the rotational energy transfer in molecular systems.

According to the power-gap "law"

$$
\sigma\left(j_{\mathrm{i}} \rightarrow j_{\mathrm{f}}\right)=a\left(2 j_{\mathrm{f}}+1\right)\left(T_{\mathrm{f}} / T_{\mathrm{i}}\right)^{1 / 2}|\Delta E|^{-\gamma},
$$
India.

*Present address: Department of Physics, Government Polytechnic, Dhar (M.P.), 454001, 
where $a$ and $\gamma$ are the fitting parameters that depend on the system and its energy, $T_{\mathrm{f}}$ and $T_{\mathrm{i}}$ are final and initial translational energies, respectively, $j_{\mathrm{i}}$ and $j_{\mathrm{f}}$ are the initial and final rotational quantum numbers, and $|\Delta E|$ is the energy gap between initial and final rotational levels.

The dependence of $a$ and $\gamma$ on $|\Delta E|$ was first pointed out by Noor Batcha and Sathyamurthy [6]. By analysing ten sets of data on $\mathrm{CO}_{2}-\mathrm{H}_{2}$ system they found that for $|\Delta E| \leq|\Delta E|^{*}$ one set of parameters $a_{\text {low }}$ and $\gamma_{\text {low }}$ and another set of parameters $a_{\text {high }}$ and $\gamma_{\text {high }}$ for $|\Delta E|>|\Delta E|^{*}$ are needed to fit the data. Several subsequent studies [7-9] observed the existence of such two regions. Schinke [9] pointed out that the transitions with $|\Delta E| \leq|\Delta E|^{*}$ correspond to the classically "allowed" region and those with $|\Delta E|>|\Delta E|^{*}$ correspond to the classically "forbidden" region. It is customary to identify $|\Delta E|^{*}$ as the maximum classical limit of rotational energy transfer.

The maximum classical limit of rotational energy transfer can also be obtained using "hard ellipsoid model" $[10,11]$. This model treats a molecule as a hard ellipsoid and the collision between an atom and a molecule is considered equivalent to the collision between the atom and the hard ellipsoid. It has been observed $[12,13]$ that for a large number of systems an energetically open channel is closed due to the angular momentum conservation constraints. In recent years, there have been several studies $[11,14]$ to obtain the classical limit of rotational energy transfer, $(\Delta E)_{\max }$, by using the angular momentum conservation constraints along with the hard ellipsoid model.

In view of the above-mentioned studies it becomes interesting to compare $|\Delta E|^{*}$ so obtained by using the PG law and $(\Delta E)_{\max }$ given by the hard ellipsoid model. In this paper we shall see that they are comparable. Further, we shall discuss that such equivalence of $|\Delta E|^{*}$ and $(\Delta E)_{\max }$ not only provides the physical meaning to $|\Delta E|^{*}$ given by the RET data and the PG law but is also valuable for determination of some features of the intermolecular interaction potential from the knowledge of the RET data.

In Sec. 2 we formulate the procedure for determination of $(\Delta E)_{\max }$ and $|\Delta E|^{*}$. The results are presented and discussed in Sec. 3. The conclusions are finally summarised in Sec. 4.

\section{Formulation \\ 2.1. Determination of $(\Delta E)_{\max }$}

Bosanac [11] treated this problem in a general way by computing the angular momentum transfer as a function of the orientation of the molecule and the incoming atom. We present here a simplified treatment along those lines.

Figure 1 shows the hard ellipsoid potential seen by the incoming atom due to interaction with the diatomic molecule. The component of linear momentum tangential to the ellipsoid is not altered by the collision. Therefore, the direction of change in momentum, $\Delta p$, due to collision with the hard ellipsoid potential would be along the normal as shown by $I M$ in the figure. For such a change in the linear momentum the magnitude of the conversion of orbital angular momentum of the system into the angular momentum of the diatomic molecule would be

$$
\Delta \boldsymbol{J}=b_{\mathrm{n}}|\Delta p|
$$




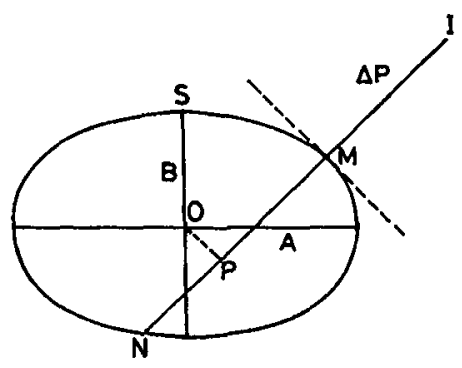

Fig. 1. A hard ellipsoid model of the rotationally inelastic atom-diatom collision.

where $b_{\mathrm{n}}$ is the length of perpendicular $O P$ on the normal $M N$. Further, for maximum possible value of $\Delta p$ one can write

$$
|\Delta p|_{\max }=p-\left(-p^{\prime}\right)=2 \sqrt{\mu}\left(\sqrt{E}+\sqrt{E^{\prime}}\right),
$$

where $\mu$ is the reduced mass of the colliding system, and $E$ and $E^{\prime}$ are the initial and final translational energies of the system, respectively. Such extreme case of maximum momentum transfer would arise when the linear momentum, $p^{\prime}$, of the scattered atom is along the normal and is in the direction opposite to the incident momentum, $p$.

For the maximum possible value of $b_{n}$ one can write the following equation by using the properties of an ellipsoid:

$$
\left(b_{\mathrm{n}}\right)_{\max }=A-B
$$

here $A$ and $B$ are lengths of the semimajor and semiminor axes, respectively.

Equations (2) to (4) yield

$$
(\Delta J)_{\max }=2 \sqrt{\mu}\left(\sqrt{E}+\sqrt{E^{\prime}}\right)(A-B) \text {. }
$$

The above expression can be used to obtain the limit of rotational energy transfer in the molecule. For a simplest case, let us consider the diatomic molecule initially in the ground state. The expression for the maximum amount of rotational energy transfer would be

$$
(\Delta E)_{\max }=(\Delta J)_{\max }^{2} / 2 I=(\mu / I)(A-B)^{2}\left(E+E^{\prime}+2 \sqrt{E E^{\prime}}\right)
$$

where $I$ is the moment of inertia of the diatomic molecule.

Equation (6) together with the following energy conservation equation:

$$
E^{\prime}=E-(\Delta E)_{\max }
$$

can be used to compute $(\Delta E)_{\max }$ from the knowledge of $E, A, B, \mu$ and $I$.

\subsection{Determination of $|\Delta E|^{*}$}

This parameter is determined with the help of cross-sections obtained from scattering calculations and the power-gap law.

For the computation of cross-sections the diatomic molecule, $\mathrm{N}_{2}$, is treated as a rigid rotor and the interaction between the molecule and the atom, $\mathrm{He}$, is taken as a pairwise sum of the spherical terms, i.e.,

$$
V=V\left(r_{1}\right)+V\left(r_{2}\right)
$$


where $r_{1}$ and $r_{2}$ are the $\mathrm{N}^{(1)}-\mathrm{He}$ and $\mathrm{N}^{(2)}-\mathrm{He}$ distances, respectively, as shown in Fig. 2.

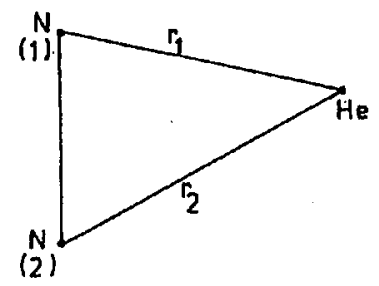

Fig. 2. Coordinates for the rigid rotor $\mathrm{N}_{2}-\mathrm{He}$ system.

TABLE I

The comparison of maximum amount of rotational energy transfer by scattering results with those predicted by the ellipsoid potential for $\mathrm{N}_{2}$ - He system with potential $V(12,6)$.

\begin{tabular}{c|c|c|c|c}
\hline \hline \multirow{2}{*}{$\begin{array}{c}\text { Energy } \\
{[\mathrm{eV}]}\end{array}$} & $\gamma_{\text {low }}^{a}$ & $\gamma_{\text {high }}^{a}$ & \multicolumn{2}{|c}{$(\Delta E)_{\max }[\mathrm{eV}]$} \\
\cline { 4 - 5 } & & & Scattering & $\begin{array}{c}\text { Ellipsoid } \\
\text { model }\end{array}$ \\
\hline 0.15 & 0.805 & 8.10 & 0.0488 & 0.0475 \\
0.25 & 0.808 & 8.78 & 0.0797 & 0.0800 \\
0.35 & 0.833 & 9.25 & 0.1130 & 0.1131 \\
0.45 & 0.809 & 7.92 & 0.1350 & 0.1466 \\
0.55 & 0.802 & 6.65 & 0.1570 & 0.1803 \\
\hline
\end{tabular}

${ }^{a}$ The units of $\gamma_{\text {low }}$ and $\gamma_{\text {high }}$ are such that in Eq. (1) cross-section is in $\AA^{2}$ and $\Delta E$ is in $\mathrm{eV}$.

TABLE II

Comparison of $A-B$ values in $\AA$ given by the potential, $V(18,6)$, and those predicted by the scattering data for $\mathrm{N}_{2}-\mathrm{He}$ system.

\begin{tabular}{c|c|c|c|c}
\hline \hline \multirow{2}{*}{$\begin{array}{c}\text { Energy } \\
{[\mathrm{eV}]}\end{array}$} & \multirow{2}{*}{$\gamma_{\text {low }}$} & \multirow{2}{*}{$\gamma_{\text {high }}$} & \multicolumn{2}{|c}{$A-B$} \\
\cline { 4 - 5 } & & & Predicted & Potential \\
\hline 0.15 & 0.809 & 8.45 & 0.539 & 0.502 \\
0.25 & 0.775 & 9.34 & 0.526 & 0.504 \\
0.35 & 0.723 & 8.19 & 0.493 & 0.505 \\
0.45 & 0.711 & 6.80 & 0.472 & 0.507 \\
0.55 & 0.711 & 5.42 & 0.458 & 0.508
\end{tabular}


For $V\left(r_{i}\right)$ the following general form of $L-J$ potential is taken:

$$
V\left(r_{i}\right)=\varepsilon\left\{[m /(n-m)]\left(r_{0} / r_{i}\right)^{n}-[n /(n-m)]\left(r_{0} / r_{i}\right)^{m}\right\}, \quad i=1,2,
$$

where $r_{0}$ and $\varepsilon$ are taken [15] as $3.60 \AA$ and $2.328 \mathrm{meV}$ respectively for $\mathrm{N}_{2}-\mathrm{He}$ system.

The results shown in Table I are obtained for potential, $V(12,6)$, which is obtained by putting $n=12$ and $m=6$ in Eq. (9). While the results shown in Table II are obtained for potential, $V(18,6)$, which is obtained by putting $n=18$ and $m=6$ in Eq. (9).

The cross-sections were computed by using Agrawal and Raff's modified version [16] of the infinite order sudden approximation. The phase shifts were computed using a 10-point Gauss-Mehler quadrature of the WKB phase shift equation as described by Pack [17].

The power-gap "law" given by Eq. (1) yields the following equation:

$$
Y=-\gamma X+\ln a,
$$

where

$$
Y=\ln \left[\sigma\left(j_{\mathrm{i}} \rightarrow j_{\mathrm{f}}\right)\left(T_{\mathrm{i}} / T_{\mathrm{f}}\right)^{1 / 2} /\left(2 j_{\mathrm{f}}+1\right)\right],
$$

and

$$
X=\ln |\Delta E| .
$$

Figure 3 gives typical $X-Y$ plot for $\mathrm{N}_{2}-\mathrm{He}$ system at initial translational energy of $0.15 \mathrm{eV}$ for the potential given by Eq. (9) with $n=12$ and $m=6$. This plot shows the existence of two straight lines signifying the two regions. The

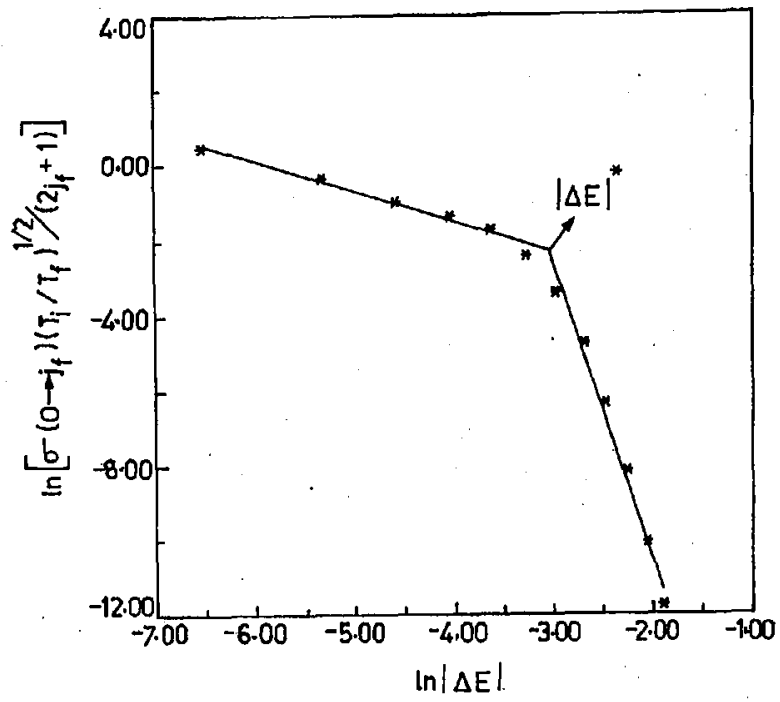

Fig. 3. $\ln \left[\sigma\left(T_{\mathrm{i}} / T_{\mathrm{f}}\right)^{1 / 2} /\left(2 j_{\mathrm{f}}+1\right)\right]$ versus $\ln |\Delta E|$ for $j_{\mathrm{i}}=0$ at $E=0.15 \mathrm{eV}$ for $V(12,6)$ potential. 
location of the critical point was marked as $|\Delta E|^{*}$ in the figure. For all sets of computed cross-sections, $|\Delta E|^{*}$ was obtained by such plots.

\section{Results and discussion}

For the application of relation (6) on a realistic potential energy surface, instead of the ideal case of hard ellipsoid potential, the values of $A$ and $B$ of Eq. (6) were obtained by assuming that the hard potential surface is represented by the classical turning point surface of the real potential employed in the calculation of cross-sections. It is interesting to see that such surface is very close to an ellipsoid. The lengths of the semimajor and semiminor axes of such an ellipsoid are identified as $A$ and $B$, respectively.

It is found that $A$ as well as $B$ decreases and $A-B$ increases with the increase in the collision energy. The typical values of $A$ and $B$ are 3.0328 and $2.5564 \AA$, respectively, for $V(12,6)$ potential at $E=0.15 \mathrm{eV}$. Further, $A$ decreases from 3.0328 to $2.8020 \AA$ and $A-B$ increases from 0.4764 to $0.4868 \AA$ as $E$ increases from 0.15 to $0.55 \mathrm{eV}$.

Table I lists $\gamma_{\text {low }}, \gamma_{\text {high }}$ and $|\Delta E|^{*}$ given by Eq. (6) as a function of initial translational energy for $\mathrm{N}_{2}-\mathrm{He}$ system with potential given by Eq. (9).

A comparison of the values of $(\Delta E)_{\max }$ given by Eq. (6) and $|\Delta E|^{*}$ given by scattering method and power-gap "law" shows that they are in very good agreement. This excellent agreement shows that $|\Delta E|^{*}$ can be considered as $(\Delta E)_{\max }$.

The data reported in Table $I$ also reveals that $|\Delta E|^{*}$ or $(\Delta E)_{\max }$ is approximately proportional to $E$ : $(\Delta E)_{\max } / E$ varies from 0.317 to 0.328 as $E$ increases from 0.15 to $0.55 \mathrm{eV}$. The variation of $(\Delta E)_{\max } / E$ with $E$ can be analysed by the two factors: $(A-B)^{2}$ and $\left(E+E^{\prime}+2 \sqrt{E} E^{\prime}\right) / E$ occurring in Eq. (6). The factor $(A-B)^{2}$ increases from 0.227 to 0.237 and the latter factor decreases from 3.3366 to 3.3119 as $E$ increases from 0.15 to $0.55 \mathrm{eV}$. For a perfectly hard ellipsoid potential $(A-B)^{2}$ would not depend on $E$ and as such the variation in $(\Delta E)_{\max } / E$ would be given by the latter factor only.

Equation (6) also reveals that $(\Delta E)_{\max } / E$ would increase with the increase in $\mu$. It was observed that $(\Delta E)_{\max } / E$ increases by about a factor of 2.5 as He is replaced by Ne. Thus, in general, depending on $\mu, I$, and $A-B$ there can be a large number of systems for which $(\Delta E)_{\max } / E \rightarrow 1$. For such systems, contrary to the limit of rotational energy transfer due to angular momentum constraints observed here, the energy conservation constraints would limit the angular momentum transfer.

One of the main advantages of the study of RET cross-sections is to obtain the anisotropic intermolecular potential of the system. The inversion of the experimental cross-sections to obtain the intermolecular potential has been the subject of considerable interest in the recent years [18-21]. The value of $A-B$ of Eq. (6) can be determined from the present conclusion, $(\Delta E)_{\max }=|\Delta E|^{*}$, and the analysis of scattering cross-sections by using the power-gap "law". For a comparison of $A-B$ value predicted by the scattering data, the power-gap "law" and Eq. (6) with the actual value given by the potential, the results are presented for $V(18,6)$ potential for $\mathrm{N}_{2}-\mathrm{He}$ system in the energy range 0.15 to $0.55 \mathrm{eV}$ in Table II. We see that the agreement is very good. Though such a determination of $A-B$ does not give 
the complete intermolecular potential yet it is important feature of the desired potential and can prove valuable in the determination of the potential surface.

Among the five parameters of the PG law, $a_{\text {low }}, \gamma_{\text {low }}, a_{\text {high }}$ and $\gamma_{\text {high }}$ and $|\Delta E|^{*}$ the present studies provide the physical significance of only one parameter, $|\Delta E|^{*}$. The validity of the hard ellipsoid model seen here also provides an understanding that the conversion of orbital angular momentum into the angular momentum of the molecule takes place in the region close to the classical turning point. Likewise, one may expect that the further research in this area may lead to the physical explanation of other four parameters of the PG law and a better understanding of the mechanism of the rotational energy transfer in the molecular systems.

\section{Summary}

The classical limit of maximum rotational energy transfer was reviewed for a hard ellipsoid potential model. The conversion of orbital angular momentum into the angular momentum of the diatomic molecule was considered at the hard wall only.

The $|\Delta E|^{*}$ values obtained by scattering results and the two-parameter power-gap "law" are compared with those given by $(\Delta E)_{\max }$ values predicted by the hard ellipsoid model. The good agreement between $|\Delta E|^{*}$ and $(\Delta E)_{\max }$ over a wide range of energies and different potential functions suggest that the parameter $|\Delta E|^{*}$, given by PG "law" has a physical significance: $|\Delta E|^{*}$ is nothing but the classical limit of the rotational energy transfer. Further, the equivalence of $|\Delta E|^{*}$ and $(\Delta E)_{\max }$ also suggest that the classical limit of angular momentum transfer given by the hard ellipsoid potential model is meaningful even for the cross-sections computed on the real potentials provided the classical turning point surface of the soft potential is assumed as the hard potential surface.

Such a study also leads to the feasibility of obtaining the difference of semimajor and semiminor axes, $A-B$, of the hard ellipsoid potential from the scattering data. The values of $A-B$ computed using Eq. (6), scattering data and the power-gap "law" are compared with those given by the actual potential. The agreement is found to be very good.

\section{Acknowledgment}

S.T. acknowledges the award of Junior Research Fellowship by C.S.I.R., New Delhi, India, for pursuing this research.

\section{References}

[1] T.A. Brunner, R.D. Driver, N. Smith, D.E. Pritchard, Phys. Rev. Lett. 41, 856 (1978).

[2] M. Waigner, I. Al-Agil, T.A. Brunner, A.W. Karp, N. Smith, D.E. Pritchard, J. Chem. Phys. 71, 1977 (1979).

[3] T.A. Brunner, R.D. Driver, N. Smith, D.E. Pritchard, J. Chem. Phys. 70, 4155 (1979).

[4] D.E. Pritchard, N. Smith, R.D. Driver, T.A. Brunner, J. Chem. Phys. 70, 2115 (1979). 
[5] T.A. Brunner, N. Smith, A.W. Karp, D.E. Pritchard, J. Chem. Phys. 74, 3324 (1981).

[6] I. Noor Batcha, N. Sathyamurthy, Chem. Phys. Lett. 79, 264 (1981).

[7] K. Koura, J. Chem. Phys. 77, 5141 (1982).

[8] E.F. Jendrek, M.M. Alexander, J. Chem. Phys. 72, 6452 (1980).

[9] R. Schinke, J. Chem. Phys. 75, 5205. (1981).

[10] D. Beck, U. Ross, W. Schepper, Z. Phys. A 293, 107 (1979).

[11] S. Bosanac, Phys. Rev. A 22, 2617 (1980).

[12] S.L. Dexheimer, M. Durand, T.A. Brunner, D.E. Pritchard, J. Chem. Phys. 76, 4996 (1982).

[13] P.M. Agrawal, N.C. Agrawal, V. Garg, J. Chem. Phys. 83, 4444 (1985).

[14] F.A. Gianturco, J.P. Toennies, M. Bernardi, J. Chem. Soc. Faraday Trans. 87, 31 (1991).

[15] (a) American Institute of Physics Handbook, Ed. D.E. Gray, McGraw Hill, New York 1957, p. 4.129. The geometric mean rule for $\varepsilon$ and the arithmatic mean rule for $\sigma$ have been used. (b) R.A. Svelha, NASA Tech. Rep. TRR-132, 1962.

[16] P.M. Agrawal, L.M. Raff, J. Chem. Phys. 74, 3292 (1981).

[17] R.T. Pack, J. Chem. Phys. 60, 633 (1974).

[18] J.C. Belchior, J.N. Murell, S.D. Bosanac, Chem. Phys. 176, 155 (1993).

[19] S.D. Bosanac, N. Petrovic, Phys. Rev. A 41, 5909 (1990).

[20] S.D. Bosanac, J.N. Murell, J. Chem. Phys. 94, 1167 (1991).

[21]. J.C. Belchior, J.N. Murell, J. Chem. Phys. 101, 2016 (1994). 